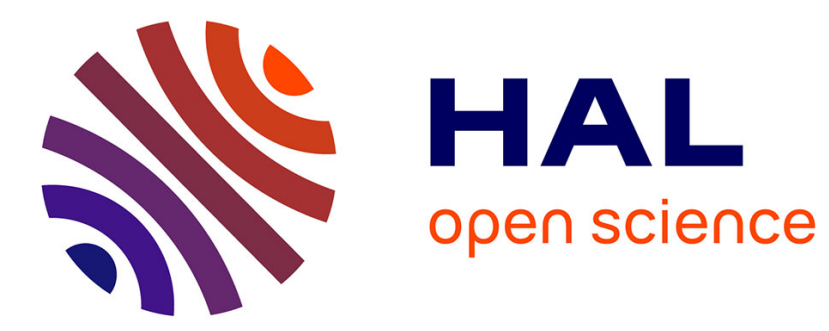

\title{
Distance-aware relay selection in an energy-efficient discovery protocol for 5G D2D communication
}

Cesar Vargas Anamuro, Nadège Varsier, Jean Schwoerer, Xavier Lagrange

\section{To cite this version:}

Cesar Vargas Anamuro, Nadège Varsier, Jean Schwoerer, Xavier Lagrange. Distance-aware relay selection in an energy-efficient discovery protocol for 5G D2D communication. IEEE Transactions on Wireless Communications, 2021, 20 (7), pp.4379 - 4391. 10.1109/TWC.2021.3058636 . hal-03160591

\section{HAL Id: hal-03160591 \\ https://imt-atlantique.hal.science/hal-03160591}

Submitted on 3 Aug 2021

HAL is a multi-disciplinary open access archive for the deposit and dissemination of scientific research documents, whether they are published or not. The documents may come from teaching and research institutions in France or abroad, or from public or private research centers.
L'archive ouverte pluridisciplinaire HAL, est destinée au dépôt et à la diffusion de documents scientifiques de niveau recherche, publiés ou non, émanant des établissements d'enseignement et de recherche français ou étrangers, des laboratoires publics ou privés. 


\title{
Distance-aware relay selection in an energy-efficient discovery protocol for 5G D2D communication
}

\author{
Cesar Vargas Anamuro, Nadège Varsier, Jean Schwoerer, and Xavier Lagrange
}

\begin{abstract}
Massive machine-type communications (mMTC) is one of the main services delivered by the fifth Generation (5G) mobile network. The traditional cellular architecture where all devices connect to the base station is not energy efficient. For this reason, the use of device-to-device (D2D) communications is considered to reduce the energy consumption of mMTC devices. The main idea is to use nearby user equipment (UE) as a relay and establish with it D2D communication. However, the relay selection process also consumes energy, and this consumption can be significant compared to the energy consumed during the data transmission phase.

In this paper, we propose a distributed energy-efficient D2D relaying mechanism for mMTC applications. This mechanism favors the selection of the UEs with low path loss with the mMTC device. Through mathematical analysis and simulations, we show that our mechanism allows a reduction of the total energy consumption of mMTC devices (up to $75 \%$ compared to direct transmission) when they have an unfavorable link budget. Moreover, our mechanism achieves almost constant energy consumption for a large range of $\mathrm{UE}$ densities and distances between the mMTC device and the base station.
\end{abstract}

Index Terms-D2D relaying, mMTC, energy-efficient, relay selection, stochastic geometry.

\section{INTRODUCTION}

M ASSIVE machine-type communications (mMTC) is one of the main services supported by the fifth Generation (5G) mobile network [1]. These services are characterized by a massive number of energy-constrained and low complexity devices that sporadically transmit small data packets. mMTC devices, also known as machine-type devices (MTDs), are usually located in poor coverage areas, like deep indoors or basements. Some MTDs receive downlink signals but cannot successfully transmit uplink signals. This imbalance between downlink and uplink is due to the difference between the transmission power of the base station (BS) and the transmission power of the MTD. The main challenge in mMTC applications is, therefore, to achieve energy-efficient uplink connectivity for MTDs, taking into account specific mMTC constraints [2].

Device-to-device (D2D) communication is a promising technology to improve the performance of cellular networks. It is defined as direct communication between two nearby devices without routing data through a BS [3]. This technology has been proposed for applications such as public safety services [4], extending network coverage [5], offloading network [6], [7], D2D relaying [8]-[15], etc.

C. Vargas Anamuro, N. Varsier, and J. Schwoerer are with Orange Labs, 28 Chemin du Vieux Chêne, 38240 Meylan, France (e-mail: cesaraugusto.vargasanamuro@orange.com; nadege.varsier@orange.com; jean.schwoerer@orange.com).

X. Lagrange is with the ADOPNET Team, IMT Atlantique/IRISA, 35576 Cesson-Sevigne, France (e-mail: xavier.lagrange@imt-atlantique.fr).
$\mathrm{D} 2 \mathrm{D}$ relaying is a technique that consists of using a device as a relay to help a source to communicate with its destination. In [12], the authors suggest the use of smartphones as relays for communication between users in case of natural disasters. The authors of [13], [14] propose the use of user equipments (UEs) to aggregate and relay the data from nearby MTDs. The advantage of this approach is that UEs form a virtual cellular infrastructure allowing spatial diversity to be exploited since the MTD can choose when establishing a link between the BS or a nearby UE as a relay [16]. D2D relaying can be implemented in half-duplex (HD) or fullduplex (FD). In HD the relay receives and transmits signals using orthogonal resources, while in FD the relay transmits and receives simultaneously using the same resources. FD is efficient when the data is divided in a set of packets because the relay can transmit a previously received packet to the BS while receiving a new one from the MTD. However, one of the main challenges of FD is the self-interference mitigation, which limits the practical implementation to communications with fixed infrastructure-based relays, low transmission power, and short communication ranges [17].

The D2D relaying mechanism consists of two phases [18]: the discovery phase and the data transmission phase. While in the discovery phase the MTD searches for and selects a UE that will be used as a relay, in the data transmission phase the MTD transmits its data to the relay. The relay selection can be performed in a centralized manner [11], [19], [20] or distributed manner [21], [22]. With the centralized approach, the BS selects the relay; whereas, with the distributed approach, it is the MTD itself that selects the relay that it will use.

There are multiple criteria to select a relay depending on the metrics to improve: the transmission rate [23], energy consumption [24], and the network lifetime [24], [25]. Reducing energy consumption is fundamental to address the requirements of mMTC applications. In the literature, the majority of authors do not consider energy consumption in the discovery phase since they assume that the energy consumed in the discovery phase is insignificant compared to the energy consumed in the data transmission phase [11], [15], [21]. This hypothesis can be valid when the association between the MTD and the relay is stable and when a large amount of data is transmitted. However, in mMTC applications, the MTDs transmit small data packets consuming a small amount of energy in the data transmission process. Therefore, the energy consumption in the discovery phase becomes no longer negligible [18]: it might even be equivalent to the energy consumption in the data transmission phase. Therefore, for mMTC applications the discovery phase is critical since it 
must be designed to optimize the MTD energy consumption in the data transmission phase while consuming the minimum possible amount of energy.

\section{A. Related Work}

In the literature, there are few studies that propose an energy-efficient discovery process for mMTC applications. The authors of [11] propose an energy-efficient relay selection approach based on the double auction theory in cooperative cellular networks. The authors of [14] propose that UEs aggregate data from MTDs making use of both licensed and unlicensed bands to support a greater number of devices. An optimization problem is formulated to maximize the number of scheduled MTDs with the lowest energy consumption. In [24] the authors consider that the relays are deployed by the operator and study the relay positioning problem to minimize the global energy consumption satisfying the quality-of-service (QoS) constraints of MTDs. The authors of [26] propose an optimal solution for the energy-efficient issue using a joint optimization problem of relay selection, channel allocation, and power control. All these studies consider a centralized approach to optimize energy consumption. The advantage of this approach is that the optimal relay is easily selected by the BS. However, the BS needs to know all the information about the MTD-UE channel states, which increases the signaling overhead between the MTDs and the BS during the discovery phase. The latter can turn into a major problem, especially for mMTC applications [27]. This justifies the research on distributed approaches with moderate signalling.

In [28], the authors of our team propose a distributed relay selection protocol for sensor networks: the node with the smallest path loss with respect to the sink has a higher probability for being selected as a relay. The authors extended their research by adding the residual energy of the nodes relay as a parameter of the next-hop node selection in [25]. They show that their protocol extends the network lifetime, reduces the average number of hops, and the average End-to-End delay. The authors of [21] propose two relay selection schemes: (i) one to minimize the overall energy consumption and (ii) one to maximize the network lifetime. Both schemes use the Requestto-Send and Clear-to-Send (RTS/CTS) handshake to obtain channel gains. Based on this information, the relay candidate that minimizes the total energy consumption has the highest probability to win the contention process. However, the authors do not consider the extra energy consumption due to the RTS/CTS transmission and the relay competition. These previous studies are focused on wireless sensor networks, where all devices including those serving as relays have strict energy consumption constraints. Furthermore, the results presented in these related studies were obtained only by simulations. The authors of [18] propose a decentralized mechanism to select the best relay using a splitting-based algorithm. In this mechanism, the total number of potential relays participating in the contention process should be known by the potential relays. However, this parameter is difficult to determine in scenarios where UEs play the role of relays due to the mobility of users.
In the literature, there is no proposal of a relay-selection distributed protocol with moderate signalling together with an analytical and therefore reproducible study.

\section{B. Objective}

Our objective is to reduce the energy consumption of MTDs with an unfavorable uplink budget. We thus propose the use of a relaying mechanism with the following design guidelines.

- The mechanism should be simple to implement.

- It should work when the MTD and/or the potential relays are mobile. Thus, it should not use the knowledge of the environment (e.g. the number of potential relays) except what can be directly measured by the MTD or the UEs.

- The mechanism should be efficient for short packets (up to 1000 bytes). The objective is not to minimize the energy consumption during the data transmission phase but to minimize the total consumption of the MTD during both the discovery and the data transmission phases.

- The target is not to systematically select the relay that has the lowest path loss with the device but only to favor relays with low path loss from a statistical point of view.

In this paper, we propose a relaying mechanism that allows a reduction of the total MTD energy consumption. To reduce MTD energy consumption in the discovery phase, the MTD sends only one discovery message and waits for the response from a relay candidate (UE located close to the MTD). Once the UEs receive the discovery message, they randomly choose a time-slot from a fixed-size contention window to respond to the MTD. We already explored this approach in [10]. The novelty of this paper lies in the time-slot selection, based on a truncated geometric distribution, which has the MTD-UE path loss as one of its parameters. This allows our protocol to prioritize the selection of the UE with the lowest MTDUE path loss without wasting energy looking exactly for the best UE. It is well established that the lower the MTD-relay path loss, the lower the MTD energy consumption during the data transmission phase. In other words, our protocol provides an acceptable compromise between energy consumption in the discovery phase and selecting the best relay for the data transmission phase.

Another benefit of selecting the UE with the lowest MTDUE path loss is that the D2D communication is short and the same resources can therefore be reused for other D2D links, reducing radio resource consumption within the cell. On the other hand, the drawback of this configuration is that the UE cannot work in full-duplex mode as it is far from the BS, and thus requires a high transmission power. Therefore, in this paper, we consider that MTDs and relaying UEs work in half-duplex mode.

\section{Key Contributions}

The main contributions of this work can be summarized as follows:

1) We propose a distributed access and relay selection mechanism, which gives priority to nearby UEs to be selected as relays. This mechanism is suitable for mMTC 


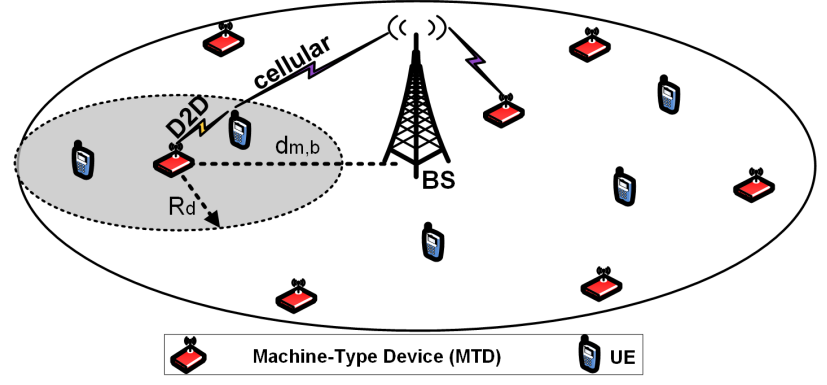

Fig. 1. Network model: UEs establish direct communication with the BS, whereas an MTD can choose between transmitting directly to the BS (cellular mode) and using a nearby UE as a relay (D2D mode).

applications since it is efficient in terms of MTD energy consumption and can easily be implemented. Our mechanism achieves almost constant energy consumption for a large range of UE densities and MTD-BS distances.

2) Using stochastic geometry, we provide an analytical framework to analyze the performance of our mechanism in terms of relay discovery probability, the average number of time slots used in the contention process, probability density function (PDF) of the MTD-relay distance, and the average MTD energy consumption in the D2D mode. This analytical framework is based both on homogeneous and inhomogeneous Poisson point processes.

The rest of the paper is organized as follows. Section II presents the system model considered in this paper. In Section III, we present our D2D relaying mechanism and the random choice of time slot. In Section IV a detailed analysis of our mechanism is conducted. The numerical results and discussion are presented in Section V. Finally, conclusions and future work are given in Section VI.

\section{SySTEM MODEL}

\section{A. Network Model}

We consider a single cell in which the UEs and the MTDs form two independent homogeneous Poisson point process $(\mathrm{H}-$ PPP) with densities $\lambda_{u}$ and $\lambda_{m}$, respectively. As shown in Fig. 1, we assume that all the UEs and MTDs are in coverage and thus they can transmit their data directly to the BS (cellular mode). However, an MTD that is in poor coverage may use a nearby $U E$ as a relay (D2D mode) to reduce its energy consumption. We assume overlay inband D2D where the BS allocates dedicated resources for D2D links in order to mitigate interference between D2D and cellular communications.

\section{B. Propagation Model}

We model the signal propagation considering a log-normal shadowing propagation model [28]. We assume a fixed transmission power $P_{t x}$. Thus, the received power $P_{r x}$ is obtained as

$$
P_{r x}=P_{t x} K r_{g}^{-\alpha} \exp (\chi),
$$

where $r_{g}$ is the Euclidean distance between transmitter and receiver, $K$ and $\alpha$ are the path-loss factor and the path-loss exponent, respectively, and $\chi$ is a zero-mean Gaussian random variable with variance $\sigma^{2}$. Log-normal shadowing is also characterized in terms of its dB-spread $\sigma_{d B}=10 \sigma / \ln (10)$. We assume that $\chi$ is constant during the discovery phase. We also consider that each communication mode (cellular and D2D) has specific $K$ and $\alpha$ values. To simplify the analysis, we use the displacement theorem [29, lemma 1]. Thus, the initial homogeneous PPP $\Phi_{u}$ with shadowing is transformed into a homogeneous PPP $\Phi_{u}^{\prime}$ with density $\lambda_{u}^{\prime}=\lambda_{u} e^{2 \sigma^{2} / \alpha^{2}}$ without shadowing but with modified distance $r=\exp (-\chi / \alpha) r_{g}$ [30]. We can rewrite (1) as

$$
P_{r x}=P_{t x} K r^{-\alpha} \text {. }
$$

Now, we have a distance-based path loss model in (2). In the rest of this paper, the performance analysis of D2D communications (MTD-UE links) will be based on the modified distances, which combine the path loss due to the distance and the shadowing effects. We can also derive the path loss as a function of the modified distance:

$$
L=r^{\alpha} / K
$$

\section{Energy Consumption Model}

In order to compute the total MTD energy consumption, we consider a model based on energy states. In this model, an MTD can operate in one of the following states: Active (Tx or $\mathrm{Rx}$ ), or Idle. The MTD transmission power is fixed, thus each state has a specific and constant power consumption [31]. The total energy consumed by the MTD is computed as:

$$
E_{m, \text { total }}=P_{m, T} t_{m, T}+P_{m, R} t_{m, R}+P_{m, I} t_{m, I}
$$

where $P_{m, T}, P_{m, R}$, and $P_{m, I}$ are the MTD power consumption (energy consumption per unit time) in Tx state, Rx state, and Idle state, respectively; $t_{m, T}, t_{m, R}$, and $t_{m, I}$ are the proportion of time spent in $\mathrm{Tx}$ state, Rx state, and Idle state, respectively.

\section{D2D RELAYING PROTOCOL DESCRIPTION}

\section{A. Assumptions}

Before introducing our relaying mechanism, the following assumptions are made:

- The channel is reciprocal, i.e., the channel from MTD to UE is the same as the channel from UE to MTD [28].

- The MTD estimates the downlink path loss from the measurement of Reference Signal Received Power (RSRP) and the transmission power of the downlink reference signals, which is broadcast by the BS [32].

- The set of relay candidates groups all UEs that satisfy the condition $L_{m, u}<L_{t h}$, where $L_{m, u}$ is the MTDUE path loss and $L_{t h}$ is the D2D path loss threshold. The value of $L_{t h}$ can be preconfigured in advance by the cellular operator. In the transformed PPP $\Phi_{u}^{\prime}$, the condition $L_{m, u}<L_{t h}$ is equivalent to considering that a UE is a relay candidate if and only if it is located inside a circular area of radius $R_{d}$ centered around the MTD. This area is called discovery area. From (3), 
$R_{d}=\left(K_{d} L_{t h}\right)^{1 / \alpha_{d}}$, where $K_{d}$ and $\alpha_{d}$ are respectively the path loss factor and path loss exponent for D2D links.

- We assume half-duplex operations. A destructive collision occurs when multiple devices transmit in the same time slot [13].

\section{B. D2D Relaying Mechanism}

We represent the D2D relaying procedures for MTDs and UEs in Fig. 2. These procedures consist of the following steps:

1) Synchronization and network configuration: The MTD synchronizes with the BS and reads the network configuration messages. The MTD measures the data size $D$ and the MTD-BS path loss $L_{m, b}$, and starts the discovery phase if and only if $L_{m, b}>L_{t h, c e l l}$ and $D>D_{t h, \text { cell }}$; otherwise, it transmits its data directly to the BS. $L_{t h, \text { cell }}$ and $D_{t h, \text { cell }}$ are two parameters that determine the transmission mode and are set by the cellular operator to avoid unnecessarily using a relay mechanism, e.g., in case the MTD-BS link has very low path loss.

2) RR packet transmission: The MTD broadcasts a Request-for-Relay (RR) packet that carries its ID. Then, it switches from $\mathrm{Tx}$ state to $\mathrm{Rx}$ state and waits for a response from a UE.

3) Contention process: A UE that receives an RR packet measures the MTD-UE path loss $L_{m, u}$. Only the UEs having $L_{m, u}<L_{t h}$ participate in the contention process (relay candidates). Let $W$ be the contention window size, which can be previously defined by the cellular operator. Each relay candidate chooses randomly a timeslot $s \in[1, W]$ with a probability $\rho$ and responds with a Relay-Candidate (RC) packet in that time-slot. The RC packet carries the ID of the relay candidate.

4) Feedback transmission: The first relay candidate that transmits an RC packet without collision wins the contention process and will be the selected relay. The MTD broadcasts a feedback packet as soon as it successfully receives an $\mathrm{RC}$ packet. In this packet, the MTD announces the selected relay ID and the modulation and coding schemes (MCS) to be used during the data transmission phase. After reading the feedback packet, only the selected relay remains active (i.e., in Rx state).

5) Data transmission: If the MTD finds a relay, it establishes a D2D link with the selected relay to transmit its data. Otherwise, the MTD transmits directly to the BS.

To avoid collisions between the packets transmitted by the MTD and those transmitted by the relay candidates, the MTD is the only one that can transmit from the beginning of the time slot. The relay candidates first listen to the beginning of the time slot and remain in the Rx state if the MTD is transmitting.

Fig. 3 (a) shows the packet exchange sequence when the MTD selects a relay during the discovery phase. In this network, $W=6$ and there are five relay candidates. UE-1 and UE-3 respectively choose time slot 4 and 6, while UE-2 and UE-4 randomly select time slot 2 . In time slot 1 no relay candidate responds, thus the MTD waits for the next time slot.
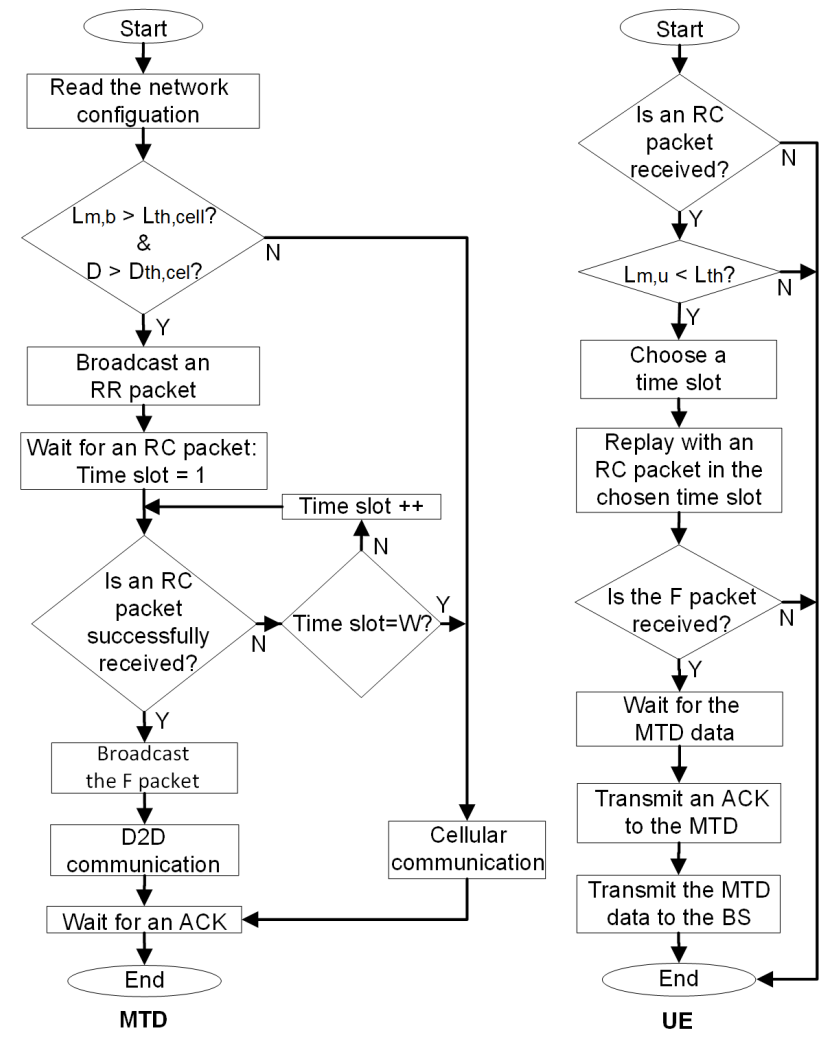

Fig. 2. D2D relaying procedure for the MTD and the relay candidates (UEs).

In time slot 2 a collision occurs, so the MTD has to wait for the next time slot. In time slot 3, only UE-5 transmits an RC packet (i.e., single slot) and thus wins the contention process. In the next time slot, the MTD broadcasts the feedback packet indicating the ID of the selected relay (in this example UE5) and forcing the other UEs to stop the contention process. Note that in time slot 4, UE-1 does not transmit since it hears that the MTD is transmitting something. Fig. 3 (b) shows the packet exchange sequence when the MTD does not select a relay. UE-1 and UE-2 select the same time slot and thus a collision occurs in time slot 2. The MTD waits for an RC packet until the maximum number of time slots $W=6$ is reached. Since the MTD does not successfully receive any $\mathrm{RC}$ packet, it transmits directly to the BS.

In [10], we have proposed a similar D2D relaying mechanism, in which the contention process was based on a uniform distribution. Unlike [10], in this paper, we propose a contention process based on a truncated geometric distribution. This improvement allows to select the nearby UEs as relays without increasing the energy consumption in the discovery phase.

\section{Random Choice of Time-slot}

According to the D2D relaying mechanism, the relay candidate that chooses the first time-slots has a higher probability of being selected as a relay. In other words, to prioritize the choice of the nearest UEs as relays, they must respond in the first time-slots. To perform this task, we consider a random choice of time-slot based on a truncated geometric distribution 

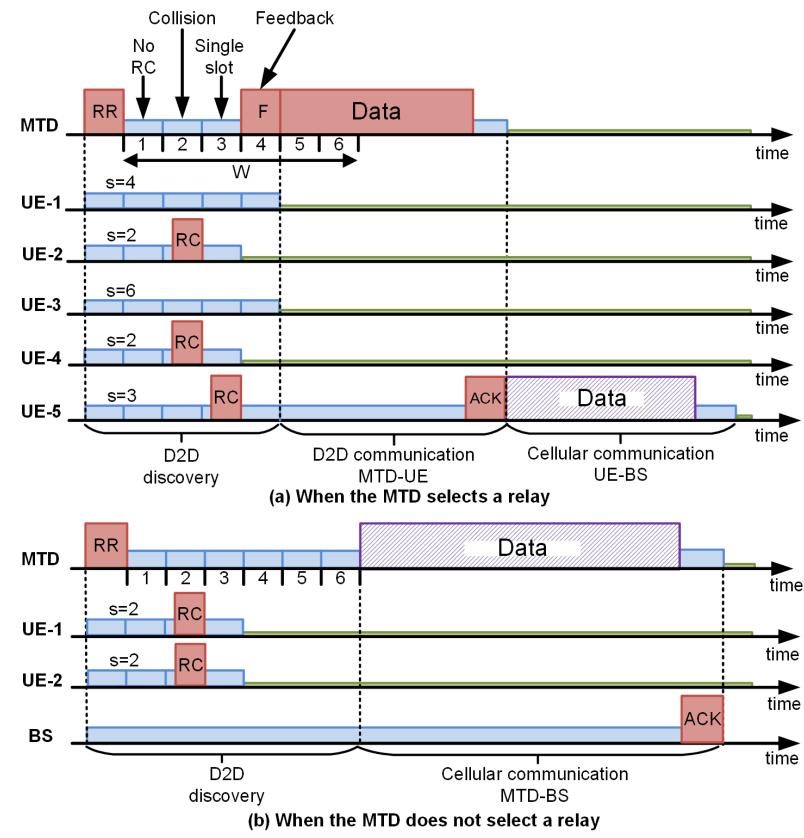

Fig. 3. Packet exchange sequence in our D2D relaying mechanism for $W=6$ with five (a) and two (b) relay candidates.

[28], where a parameter of this distribution depends on the modified MTD-UE distance.

1) Truncated geometric random choice case: The relay candidates randomly choose one time-slot $s \in[1, W]$, where $W$ is the contention window size. The probability of transmitting in time slot $s$ depends on the modified MTD-UE distance $r$ as [28]:

$$
\rho(s, r)=\frac{g(r)^{s-1}(1-g(r))}{1-g(r)^{W}}, s \in[1, W]
$$

where

$$
g(r)=b+\frac{1-b^{2}}{b}\left(\frac{r}{R_{d}}\right)
$$

where the parameter $0<b<1$ is constant and tunable to allow a UE closer to the MTD to have a higher probability of sending an RC packet. As a result, each relay candidate has a particular probability mass function (PMF) of the time slot choice. Fig. 4 shows the PMFs of the time slot choice in a scenario where an MTD is located at the origin, and there are four UEs in the discovery area. UE-1, UE-2, UE-3, and UE-4 are located 30, 120, 210, and 300 meters from the MTD, respectively. In this example, we assume $R_{d}=300$ meters, $b=0.6, W=8$ time-slots, and shadowing $\sigma_{d B}=8$ $\mathrm{dB}$.

2) Uniform random choice case: We can show that the uniform random choice of time slot employed in [10] is a particular case of the truncated geometric random choice. When $b \rightarrow 1$, from (6) we get $g(r) \rightarrow 1$ and thus in (5) we have

$$
\lim _{g(r) \rightarrow 1} \rho(s, r)=\lim _{g(r) \rightarrow 1} \frac{g(r)^{s-1}}{\sum_{k=1}^{W} g(r)^{k-1}}=\frac{1}{W} .
$$
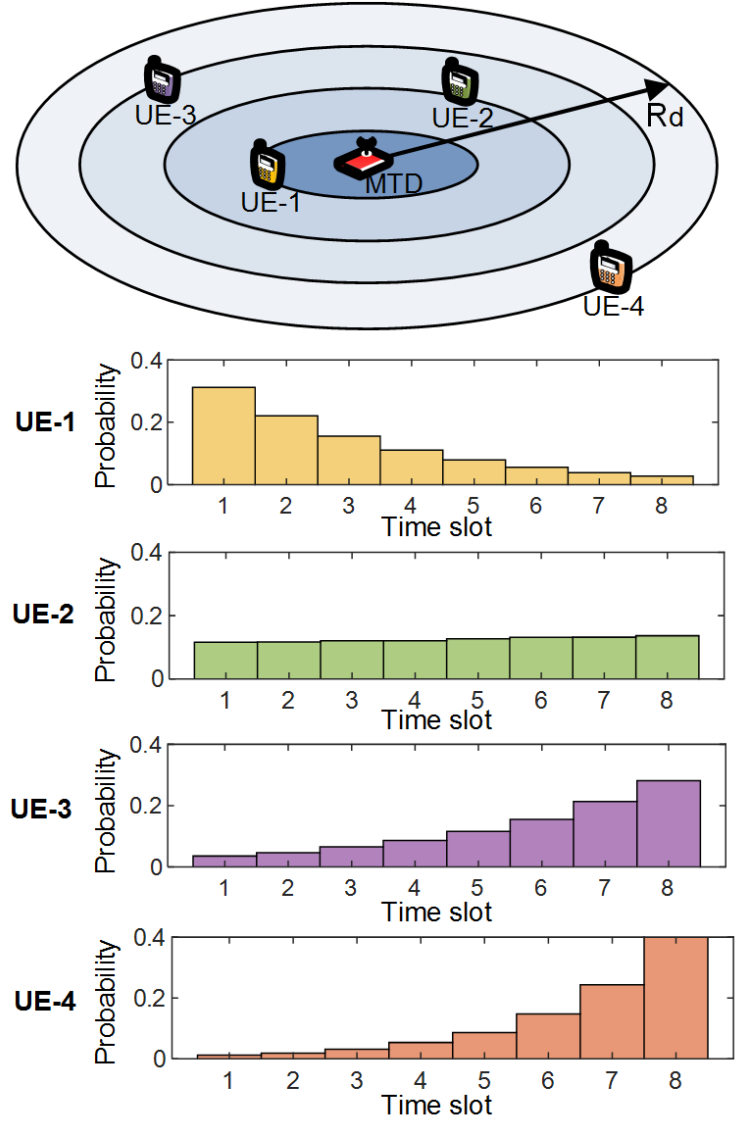

Fig. 4. PMFs of the time slot choice in a network with four relay candidates, $R_{d}=300$ meters, $b=0.6$, shadowing $\sigma_{d B}=8 \mathrm{~dB}$, and $W=8$ time-slots. UE-1, UE-2, UE-3, and UE-4 are located 30, 120, 210, and 300 meters from the MTD, respectively.

The probability of choosing time slot $s \in[1, W]$ is thus independent of the MTD-UE distance.

\section{AnAlytical Model}

In this section, we derive analytical expressions to evaluate our relaying mechanism. First, we focus on the analysis of the relay discovery probability, which refers to the probability that the MTD selects a relay during the contention process. Another indicator that we analyze is the number of time slots used in the contention process. This indicator is directly related to energy consumption and radio resource consumption during the discovery phase. The mechanism has a better performance when this indicator is small. Once the MTD has selected a relay, it can estimate the MTD-relay distance and accordingly adapts the MCS that it will use during the data transmission phase. We thus derive the PDF of the MTD-relay distance. Finally, we provide analytical expressions for the total energy consumed by the MTD in cellular mode and D2D mode. We evaluate our mechanism based on the energy model defined by 3GPP in [31]. The baseline scenario consists of an MTD that sends its data to the BS through cellular communication.

\section{A. Relay Discovery Probability}

At a given time slot $s$, all the relay candidates located at distance $r$ from the MTD have the same probability $\rho(s, r)$ of 
transmitting an RC packet (i.e., isotropic intensity function), but this probability changes depending on $r$. Thus, the relay candidates form an inhomogeneous PPP (I-PPP) centered around the MTD and with density function $\lambda(r)=\rho(s, r) \lambda_{u}^{\prime}$, where $r$ is the radius in polar coordinates, and $\rho(s, r)$ is given in (5). Using the definition of an I-PPP [33], we can deduce the probability of receiving $n \mathrm{RC}$ responses in time slot $s$ :

$$
\mathbb{P}(N=n)=\frac{\left(\Lambda_{s}\right)^{n}}{n !} \exp \left(-\Lambda_{s}\right)
$$

where $\Lambda_{s}$ is the intensity measure, which is given by

$$
\Lambda_{s}=2 \pi \int_{0}^{R_{d}} \lambda(r) r d r=2 \pi \lambda_{u}^{\prime} \int_{0}^{R_{d}} \rho(s, r) r d r .
$$

The MTD successfully receives an RC packet in time slot $s$ if only one relay candidate answers in that time slot (single time-slot). From (8) we can find the probability that time slot $s$ is a single time slot:

$$
P_{1}(s)=\mathbb{P}(N=1)=\Lambda_{s} \exp \left(-\Lambda_{s}\right) .
$$

Since in each time slot $s$ the relay candidates form an independent thinning PPP, thus the relay discovery probability can be obtained as

$$
P_{\text {disc }}=1-\prod_{s=1}^{W}\left(1-P_{1}(s)\right) .
$$

In case of a uniform random choice of time slot: let $P_{1, \text { uni }}$ and $P_{\text {disc,uni }}$ respectively be the probability of single time slot and relay discovery probability. With the substitution of (7) into (10), we have

$$
P_{1, \text { uni }}=\frac{\pi R_{d}^{2} \lambda_{u}^{\prime}}{W} \exp \left(-\frac{\pi R_{d}^{2} \lambda_{u}^{\prime}}{W}\right) .
$$

Note that $P_{1, \text { uni }}$ is independent of $s$. Thus, the relay discovery probability defined in (11) can be simplified as follows:

$$
P_{\text {disc,uni }}=1-(1-A \exp (-A))^{W}
$$

where $A=\pi R_{d}^{2} \lambda_{u}^{\prime} / W$.

\section{B. Number of Slots Used in the Contention Process}

Let $S$ be a discrete random variable that represents the number of time slots used in the contention process. The contention process ends as soon as an RC packet is successfully received by the MTD or when the maximum number of time slots $W$ is reached.

For $S=1,2, \ldots, W-1$, the probability that the contention process ends in time slot $s$ is equivalent to the probability of a reception failure in the 1 st, 2 nd, ..., $(s-1)$ th time slots and a successful reception at the $s$ th time slot. Thus, we have

$$
\mathbb{P}(S=s)=P_{1}(s) \prod_{j=1}^{s-1}\left(1-P_{1}(j)\right), s \in[1, W-1] .
$$

The number of time slots used in the contention process is $W$ when an RC packet is successfully received in time slot $W$ (the first $W-1$ time slots fail) or when all receptions fail. Thus, the probability $\mathbb{P}(S=W)$ is equivalent to the probability of a reception failure in the 1 st, 2 nd,..., $(W-1)$ th time slots:

$$
\mathbb{P}(S=W)=\prod_{j=1}^{W-1}\left(1-P_{1}(j)\right)
$$

The average number of time slots used in the contention process can be determined as

$$
\bar{S}=\sum_{s=1}^{W} s \mathbb{P}(S=s)
$$

Substituting (14) and (15) into (16), we have

$$
\bar{S}=\sum_{s=1}^{W-1} s P_{1}(s) \prod_{j=1}^{s-1}\left(1-P_{1}(j)\right)+W \prod_{j=1}^{W-1}\left(1-P_{1}(j)\right)
$$

\section{PDF of the MTD-Relay Distance}

We derive the PDF of the MTD-relay distance in case of a successful relay discovery. Let $R$ be the distance between the MTD and the relay. The cumulative distribution function (CDF) of $R$ can be calculated as

$$
F_{R}(r)=\mathbb{P}(R \leq r)=\sum_{s=1}^{W} \mathbb{P}(R \leq r \mid s) \mathbb{P}(S=s),
$$

where $0 \leq r \leq R_{d}, \mathbb{P}(S=s)$ is the probability that an $\mathrm{RC}$ packet is successfully received in time slot $s$, which is defined in (14). $\mathbb{P}(R<r \mid s)$ is the probability that the selected relay is located inside a disk of radius $r$ given that an RC packet is successfully received in time slot $s$. This probability can be derived as follows:

$$
\mathbb{P}(R \leq r \mid s)=\frac{\int_{0}^{r} 2 \pi \lambda_{u}^{\prime} \rho(s, x) x d x}{\int_{0}^{R_{d}} 2 \pi \lambda_{u}^{\prime} \rho(s, x) x d x}=\frac{\int_{0}^{r} \rho(s, x) x d x}{I_{s}},
$$

where $I_{s}=\int_{0}^{R_{d}} \rho(s, x) x d x$.

Substituting (14) and (19) into (18), we have

$$
F_{R}(r)=\sum_{s=1}^{W} \frac{\int_{0}^{r} \rho(s, x) x d x}{I_{s}} P_{1}(s) \prod_{j=1}^{s-1}\left(1-P_{1}(j)\right) .
$$

By differentiation, we obtain the PDF of the MTD-relay distance

$$
f_{R}(r)=\frac{d F_{R}(r)}{d r}=r \sum_{s=1}^{W} \frac{\rho(s, r)}{I_{s}} P_{1}(s) \prod_{j=1}^{s-1}\left(1-P_{1}(j)\right)
$$

where $0 \leq r \leq R_{d}$.

If we consider a uniform random choice of time slot in the contention process, the PDF of the MTD-relay distance becomes

$$
f_{R, \text { uni }}(r)=\frac{2 r}{R_{d}^{2}}\left(1-\left(1-P_{1, \text { uni }}(s)\right)^{W}\right), 0 \leq r \leq R_{d},
$$

where $P_{1, \text { uni }}(s)$ is defined in (12). This result has also been derived in [10]. 


\section{Analysis of the Total MTD Energy Consumption}

1) Total MTD Energy Consumption in Cellular Mode: In cellular mode, the MTD transmits its data directly to the BS as in the traditional cellular uplink. The MTD energy consumption $E_{\text {cell }}$ in cellular mode can be computed as

$$
E_{\text {cell }}=P_{m, T} D / C_{m, b},
$$

where $P_{m, T}$ is the MTD power consumption in Tx state, $D$ is the data packet size, and $C_{m, b}$ is the data rate (bits per second) in the MTD-BS link.

Let $\theta_{m, b}$ be the signal-to-noise ratio (SNR) in the MTD-BS link. The received power is derived in (1), thus the SNR is given by

$$
\theta_{m, b}=\frac{P_{m} K_{c} \exp (\chi)}{N_{0} B_{w}\left(d_{m, b}\right)^{\alpha_{c}}}
$$

where $P_{m}$ is the MTD transmit power, $K_{c}$ and $\alpha_{c}$ are respectively the path loss factor and path loss exponent for cellular links, $B_{w}$ is the bandwidth, $N_{0}$ is the noise power spectral density, and $d_{m, b}$ is the Euclidean MTD-BS distance. Recall that $\chi$ is a zero-mean Gaussian random variable.

The MTD transmits its data only if the SNR is greater than a predefined threshold $\theta_{\text {min }}$ (transmission constraint), for example $\theta_{\min }=-10 \mathrm{~dB}$ [34]. For a given MTD-BS distance $d_{m, b}$, the SNR $\theta_{m, b}$ depends on $\chi$. We can obtain therefore the minimum value $\chi_{\min }$ that satisfies the transmission constraint $\theta_{m, b} \geq \theta_{\min }$. From (24) we have

$$
\frac{P_{m} K_{c} \exp (\chi)}{N_{0} B_{w}\left(d_{m, b}\right)^{\alpha_{c}}} \geq \theta_{\min } .
$$

So the minimum value of $\chi$ as a function of $d_{m, b}$ is:

$$
\chi_{\min }=\ln \left(\frac{\theta_{\min } N_{0} B_{w}}{P_{m} K_{c}}\right)+\alpha_{c} \ln \left(d_{m, b}\right) .
$$

The MTD does not transmit its data to the BS if $\theta_{m, b}<$ $\theta_{\text {min }}$. Otherwise, it transmits its data and we assume that it can dynamically adapt its data rate according to SNR. Thus, we use a modified Shannon capacity formula proposed in [35] for computing the data rate:

$$
C_{m, b}= \begin{cases}0, & \theta_{m, b}<\theta_{\mathrm{min}}, \\ B_{\mathrm{eff}} B_{w} \log _{2}\left(1+\theta_{m, b} / \theta_{\mathrm{eff}}\right), & \theta_{m, b} \geq \theta_{\mathrm{min}},\end{cases}
$$

where $B_{\text {eff }}$ adjusts for the system bandwidth efficiency of LTE, $\theta_{\text {eff }}$ adjusts for the SNR implementation efficiency of LTE.

Observe that the transmission constraint $\left(\theta_{m, b} \geq \theta_{\min }\right)$ is equivalent to the constraint $\chi \geq \chi_{\text {min }}$ for a given $d_{m, b}$. Combining (23) and (27) we obtain the MTD energy consumption in cellular mode:

$$
E_{\text {cell }}(\chi)= \begin{cases}0, & \chi<\chi_{\text {min }} \\ \frac{P_{m, T} D}{B_{\text {eff }} B_{w} \log _{2}\left(1+\frac{P_{m} K_{c} \exp (\chi)}{N_{0} B_{w} \theta_{\mathrm{eff}}\left(d_{m, b}\right)^{\alpha_{c}}}\right)}, & \chi \geq \chi_{\text {min }}\end{cases}
$$

It is clear that if $\chi<\chi_{\min }$, the MTD does not transmit anything and therefore it does not consume energy. Thus, the average MTD energy consumption for a given $d_{m, b}$ is

$$
\bar{E}_{\text {cell }}\left(d_{m, b}\right)=\mathbb{P}\left(\chi \geq \chi_{\text {min }}\right) \int_{\chi_{\text {min }}}^{+\infty} E_{\text {cell }}(x) f_{\chi}(x) d x,
$$

where $f_{\chi}(x)=\frac{1}{\sqrt{2 \pi \sigma^{2}}} \exp \left(\frac{-x^{2}}{2 \sigma^{2}}\right)$ is the PDF of a zero-mean Gaussian random variable with variance $\sigma^{2} ; \mathbb{P}\left(\chi \geq \chi_{\min }\right)$ is the transmission probability, which can be easily derived from the complementary cumulative distribution function (CCDF) of a zero-mean Gaussian distribution. Thus we have

$$
\mathbb{P}\left(\chi \geq \chi_{\min }\right)=\frac{1}{2}-\frac{1}{2} \operatorname{erf}\left(\frac{\chi_{\min }}{\sigma \sqrt{2}}\right),
$$

where $\operatorname{erf}(x)=\frac{2}{\sqrt{\pi}} \int_{0}^{x^{2}} \exp \left(-t^{2}\right) d t$ refers to the error function.

2) Total MTD Energy Consumption in D2D Mode: The average of the total MTD energy consumption in D2D mode can be calculated as follows:

$$
\bar{E}_{\mathrm{D} 2 \mathrm{D}, \text { total }}=\bar{E}_{\mathrm{D} 2 \mathrm{D}, \mathrm{disc}}+\bar{E}_{\mathrm{dataTx}},
$$

where $\bar{E}_{\mathrm{D} 2 \mathrm{D} \text {,disc }}$ and $\bar{E}_{\text {dataTx }}$ are the average energy consumption in the discovery phase and data transmission phase, respectively. We consider a fixed data rate during the discovery phase and a dynamic data rate adaptation during the data transmission phase.

The MTD is in Tx state when it transmits the RR and feedback packets, while it is in Rx state during the contention process. Thus, the average energy consumption during the discovery phase is

$$
\bar{E}_{\mathrm{D} 2 \mathrm{D}, \mathrm{disc}}=T_{s}\left(2 P_{m, T}+\bar{S} P_{m, R}\right),
$$

where $T_{s}$ is the time slot duration, and $\bar{S}$ is the average number of slots used in the contention process, which is given by (17).

The MTD transmits its data to the selected relay (D2D communication). However, in case no relay has been found, the MTD transmits directly to the BS as in traditional cellular communications. Thus, the average energy consumption during the data transmission phase can be derived as

$$
\bar{E}_{\text {dataTx }}=P_{\text {disc }} \bar{E}_{\mathrm{D} 2 \mathrm{D}, \mathrm{comm}}+\left(1-P_{\text {disc }}\right) \bar{E}_{\text {cell }},
$$

where $P_{\text {disc }}$ is the relay discovery probability, which is obtained from (11), $\bar{E}_{\mathrm{D} 2 \mathrm{D} \text {,comm }}$ is the average energy consumption in the D2D communication, and $\bar{E}_{\text {cell }}$ is the average energy consumption in cellular mode, which is defined in (29).

We consider that during the D2D communication the MTD transmits at fixed power. Thus, to obtain the energy consumption, we follow the same procedure used to derive (23):

$$
E_{\mathrm{D} 2 \mathrm{D}, \mathrm{comm}}=\frac{P_{m, T} D}{B_{\mathrm{eff}} B_{w} \log _{2}\left(1+\frac{P_{m} K_{d} r^{-\alpha} d}{N_{0} B_{w} \theta_{\mathrm{eff}}}\right)},
$$

where $r$ is the modified MTD-relay distance, $K_{d}$ and $\alpha_{d}$ are respectively the path loss factor and path loss exponent for D2D links. The parameter $r$ is a random variable with PDF $f_{R}(r)$ given by (21). Thus, the average energy consumption in the D2D communication is:

$$
\bar{E}_{\mathrm{D} 2 \mathrm{D}, \mathrm{comm}}=\int_{0}^{R d} \frac{P_{m, T} D}{B_{\mathrm{eff}} B_{w} \log _{2}\left(1+\frac{P_{m} K_{d} r^{-\alpha_{d}}}{N_{0} B_{w} \theta_{\text {eff }}}\right)} f_{R}(r) d r .
$$


TABLE I

SIMULATION PARAMETERS

\begin{tabular}{|c|c|}
\hline Parameter & Value \\
\hline \hline MTD transmission power $\left(P_{m}\right)$ & $23 \mathrm{dBm}$ \\
\hline MTD power consumption in Tx state $\left(P_{m, T}\right)$ & $545 \mathrm{~mW}$ \\
\hline MTD power consumption in Rx state $\left(P_{m, R}\right)$ & $90 \mathrm{~mW}$ \\
\hline MTD Bandwidth $\left(B_{w}\right)$ & $180 \mathrm{kHz}$ \\
\hline Noise Power Spectrum Density $\left(N_{0}\right)$ & $-174 \mathrm{dBm} / \mathrm{Hz}$ \\
\hline Cellular path-loss exponent $\left(\alpha_{c}\right)$ & 3.67 \\
\hline Cellular path-loss factor for a distance in meters $\left(K_{c}\right)$ & 0.0070 \\
\hline D2D path-loss exponent $\left(\alpha_{d}\right)$ & 4 \\
\hline D2D path-loss factor for a distance in meters $\left(K_{d}\right)$ & 0.0173 \\
\hline Time slot duration $\left(T_{s}\right)$ & $0.5 \mathrm{~ms}$ \\
\hline Bandwidth efficiency $\left(B_{\text {eff }}\right)$ & 0.56 \\
\hline SNR efficiency $\left(\theta_{\text {eff }}\right)$ & 2 \\
\hline SNR threshold $\left(\theta_{\min }\right)$ & $-10 \mathrm{~dB}$ \\
\hline
\end{tabular}

\section{NUMERICAL RESUltS AND DISCUSSION}

In this section, we first provide simulation results to validate our analytical results obtained in the previous section. Simulations are performed using MATLAB, where each point corresponds to the average value of 5000 iterations. In each iteration, the UEs are distributed independently according to H-PPP in a circular area of radius $1.5 \mathrm{~km}$ centered around the MTD. Table I summarizes the simulation parameters. We consider the values of the power consumption in each state according to the 3GPP specifications [31]. The bandwidth efficiency and SNR efficiency values are according to the SISO channel parameters from [35].

\section{A. Determining the Radius of the Discovery Area}

In order to avoid an empty discovery area, we determine the minimum value of $R_{d}$ so that the probability of finding at least one UE in the discovery area is greater than $98 \%$, which is equivalent to $\mathbb{P}(N=0) \leq 0.02$. In the transformed PPP $\Phi_{u}^{\prime}$, the number of UEs $N$ inside the discovery area has a Poisson distribution with mean $\lambda_{u}^{\prime} \pi R_{d}^{2}$, i.e.,

$$
\mathbb{P}(N=n)=\frac{\left(\lambda_{u}^{\prime} \pi R_{d}^{2}\right)^{n}}{n !} \exp \left(-\lambda_{u}^{\prime} \pi R_{d}^{2}\right),
$$

where $\lambda_{u}^{\prime}=\lambda_{u} e^{2 \sigma^{2} / \alpha^{2}}$. Thus, we have

$$
\mathbb{P}(N=0)=\exp \left(-\lambda_{u} e^{2 \sigma^{2} / \alpha^{2}} \pi R_{d}^{2}\right) \leq 0.02 .
$$

Considering a low UE density scenario (i.e., when $\lambda_{u}=0.1 \times$ $10^{-4} \mathrm{UEs} / \mathrm{m}^{2}$ ), shadowing $\sigma_{d B}=8 \mathrm{~dB}$, and $\alpha=4$, we obtain $R_{d} \geq 286$ meters. Hence, in this work we consider $R_{d}=300$ meters.

\section{B. Analytical Model Validation}

Fig. 5 (a) and Fig. 5 (b) show respectively the relay discovery probability and the average number of time slots used in the contention process as a function of the UE density for $W=\{8,16,32\}$. In both figures the analytical and simulation results match well, this confirms the correctness of (11) and (17). Fig. 5 (a) shows that as the UE density increases, the relay discovery probability increases until it reaches a maximum and then decreases due to the number of collisions. When the UE density increases, the number of collisions increases due to the limited number of time slots

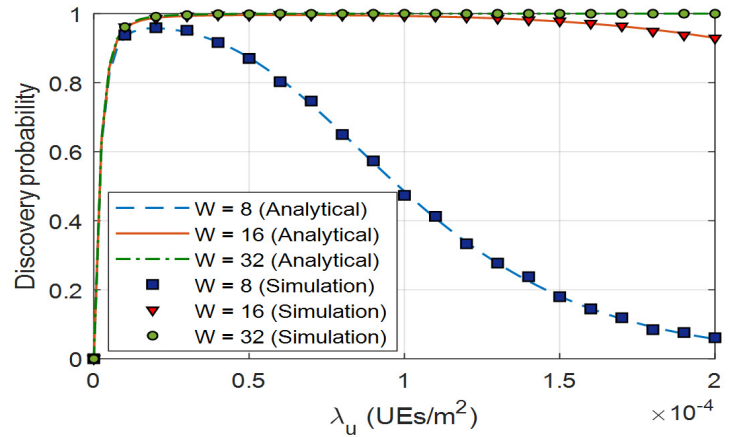

(a) Relay discovery probability

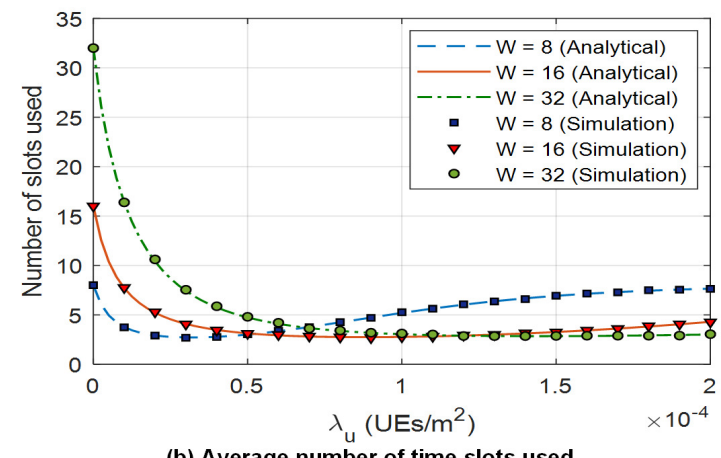

(b) Average number of time slots used

Fig. 5. Comparison of (a) the relay discovery probability and (b) the average number of time slots used in the contention process, for different $W$ values, $R_{d}=300, b=0.6$, and shadowing $\sigma_{d B}=8 \mathrm{~dB}$.

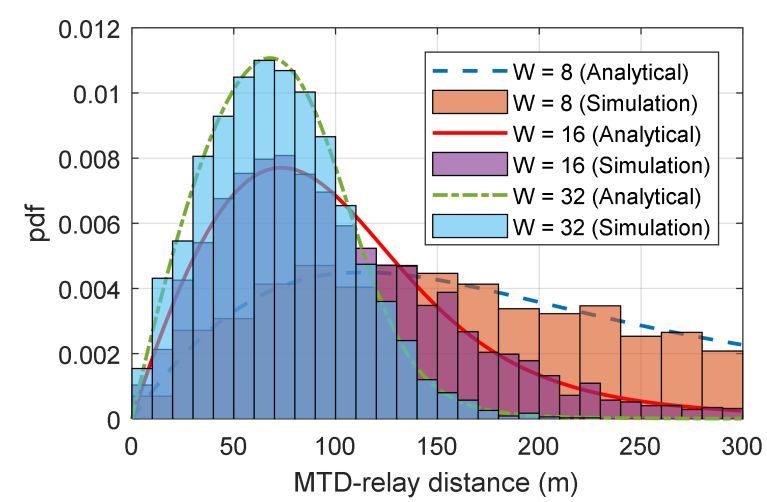

Fig. 6. Comparison of the PDF of the MTD-relay distance for different $W$ values, $R_{d}=300, b=0.6$, shadowing $\sigma_{d B}=8 \mathrm{~dB}$, and $\lambda_{u}=1 \times 10^{-4}$ $\mathrm{UEs} / \mathrm{m}^{2}$.

$(W)$. Fig. 5 (b) shows that, when the UE density is low, the MTD wastes time slots waiting for the response of a relay candidate. On the other hand, when the UE density increases the number of time slots decreases reaching a minimum value, then it increases due to the increase of the number of collisions.

Fig. 6 shows the PDF of the MTD-relay distance for $\lambda_{u}=$ $1 \times 10^{-4} \mathrm{UEs} / \mathrm{m}^{2}$, and $W=\{8,16,32\}$. From this figure, we confirm the correctness of (21). We observe that, the smaller the size of the contention window, the more probability of selecting a relay close to the MTD.

The average energy consumed by the MTD in the discovery and data transmission phases is shown in Fig. 7. The derived 


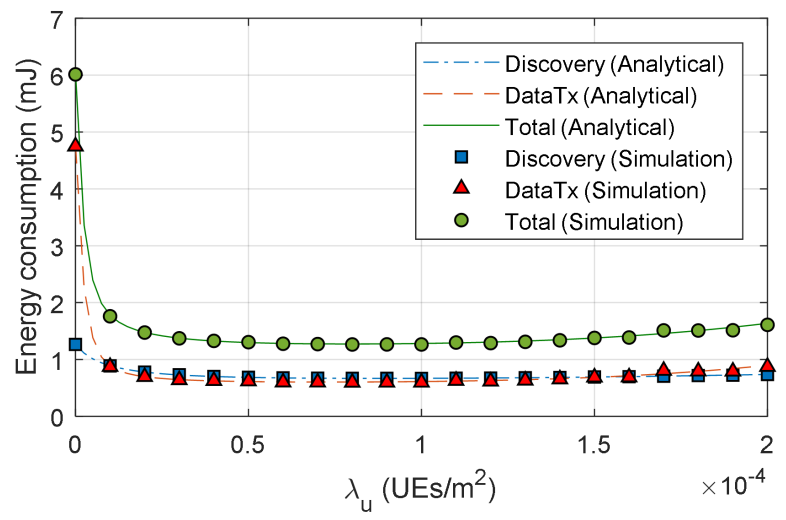

Fig. 7. Comparison between energy consumption in the discovery phase and in the data transmission phase, for $R_{d}=300, b=0.6, W=16$, shadowing $\sigma_{d B}=8 \mathrm{~dB}, D=200$ bytes, and $d_{m, b}=1000$ meters.

analytical model agrees with the simulation results well. In this figure, we consider a scenario where the MTD is located 1000 meters from the BS and it transmits 200 bytes of data. The figure shows that when $\lambda_{u} \in\left[0.1 \times 10^{-4}, 2 \times 10^{-4}\right] \mathrm{UEs} / \mathrm{m}^{2}$, as much energy is consumed in the discovery phase, as in the data transmission phase. Note that the energy consumption in the data transmission phase is very high for $\lambda_{u}=0$. The reason is that the MTD does not find a relay and thus, it transmits directly to the BS.

Since the analytical and simulation results match well, henceforth, we will only use the analytical expressions to analyze the performance of our D2D relaying mechanism.

\section{Impact of Parameter $b$}

In Fig. 8 (a), Fig. 8 (b), and Fig. 9, we analyze how the parameter $b$ affects the relay discovery probability, the average number of time slots used in the contention process, and the PDF of the MTD-relay distance, respectively. The radius of the discovery area $R_{d}$ and the contention window size $W$ are fixed to 300 meters and 16 time slots, respectively. In (7), we have shown that the uniform random choice of time-slot proposed in [10] is a particular case of our protocol when $b$ equal to 1 . Thus, we use the term Uniform when we refer to the performance of the protocol proposed in [10]. Fig. 8 (a) shows that for low UE densities $\left(\lambda_{u}<0.5 \times 10^{-4} \mathrm{UEs} / \mathrm{m}^{2}\right)$ it is better to use the uniform distribution (i.e., $b \approx 1$ ), while for high UE densities $\left(\lambda_{u}>0.5 \times 10^{-4} \mathrm{UEs} / \mathrm{m}^{2}\right)$ it is better the use of $b=0.4$ or $b=0.6$ to obtain a high relay discovery probability. We also observe that the relay discovery probability is less affected by the UE density in the range $\left[0.2 \times 10^{-4}, 2 \times 10^{-4}\right]$ if values such as $b=0.4$ and $b=0.6$ are used. Fig. 8 (b) shows that for low UE densities $\left(\lambda_{u}<0.5 \times 10^{-4} \mathrm{UEs} / \mathrm{m}^{2}\right)$, fewer time slots are used when $b \approx 1$ (uniform distribution). On the other hand, when the UE density is high $\left(\lambda_{u}>0.5 \times 10^{-4}\right.$ $\left.\mathrm{UEs} / \mathrm{m}^{2}\right)$, fewer time slots are used when $b=0.4$ and $b=0.6$.

In Fig. 9, we observe that the probability that the selected relay is close to the MTD is higher when the parameter $b$ is a small value than when it is a large value. Recall that the
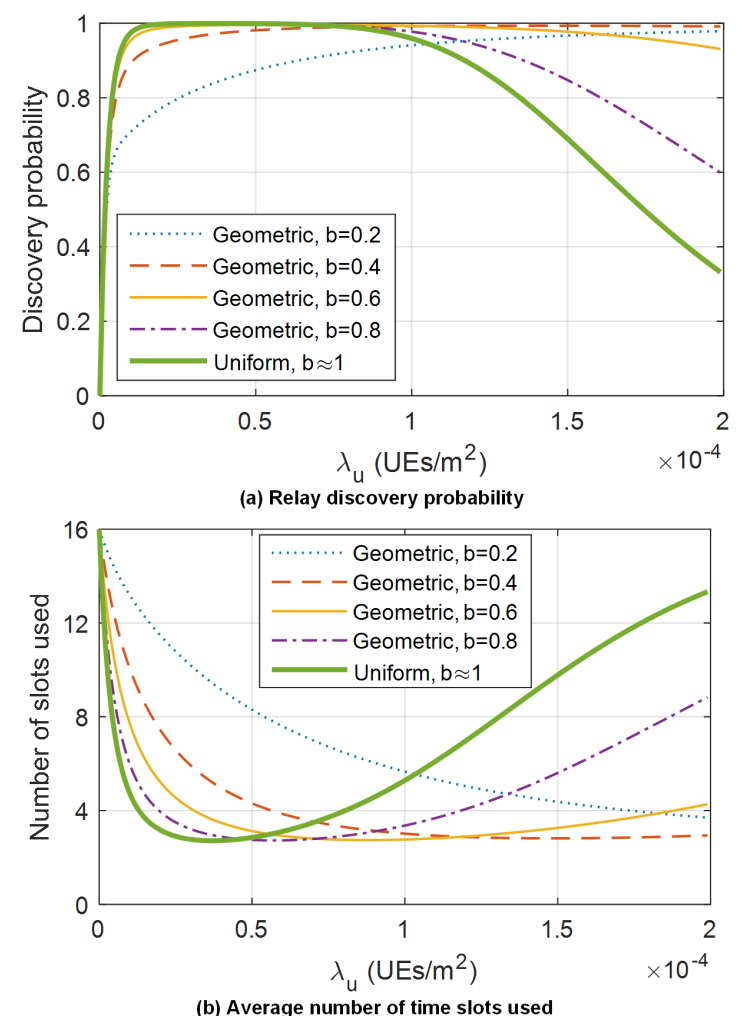

Fig. 8. Comparison of (a) the relay discovery probability and (b) the average number of time slots used during contention process, for different $b$ values, $R_{d}=300, W=16$, and shadowing $\sigma_{d B}=8 \mathrm{~dB}$.

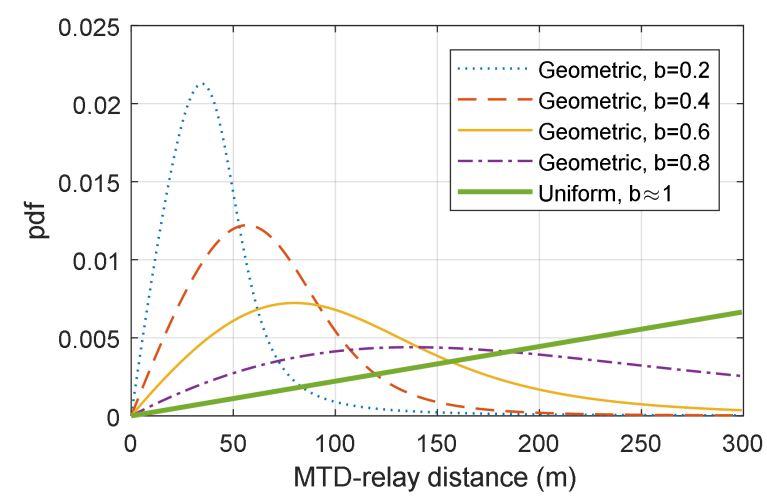

Fig. 9. Comparison of the PDF of the MTD-relay distance for different $b$ values, $R_{d}=300, W=16$, shadowing $\sigma_{d B}=8 \mathrm{~dB}$, and $\lambda_{u}=1 \times 10^{-4}$ $\mathrm{UEs} / \mathrm{m}^{2}$.

energy consumption in the data transmission phase is directly related to the MTD-relay distance.

\section{Minimization of the Total MTD Energy Consumption}

Fig. 10 shows how the parameter $b$ impacts the total MTD energy consumption for $D=200$ bytes and $d_{m, b}=1000$ meters. We can see that for $\lambda_{u}<0.8 \times 10^{-4} \mathrm{UEs} / \mathrm{m}^{2}$, parameter $b$ does not significantly affect the MTD energy consumption. However, when the UE density increases the difference in terms of total energy consumption between $b=0.4$ and $b \approx 1$ grows significantly. 


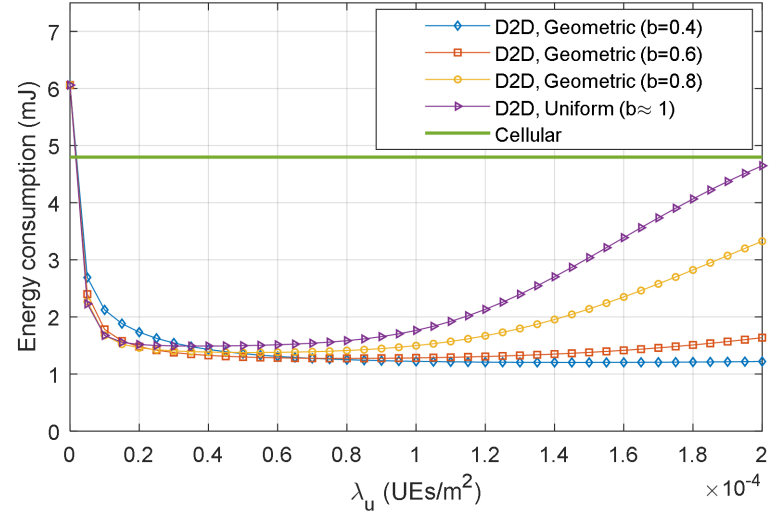

Fig. 10. Comparison of the total MTD energy consumption for different $b$ values, $R_{d}=300, W=16$, shadowing $\sigma_{d B}=8 \mathrm{~dB}, D=200$ bytes, and $d_{m, b}=1000$ meters.

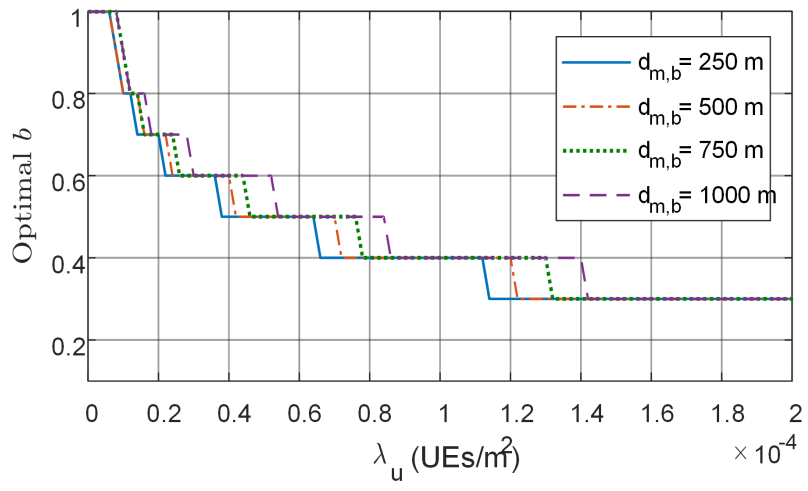

Fig. 11. Comparison of the optimal $b$ value for different UE densities and MTD-BS distances, $R_{d}=300, W=16$, shadowing $\sigma_{d B}=8 \mathrm{~dB}$, and $D=200$ bytes.

In order to determine the optimal value of the parameter $b$ minimizing the total MTD energy consumption we evaluate the energy consumption for $b=\{0.1,0.2, \ldots, 1\}$. In Fig. 11, we show the optimal value of $b$ as a function of the UE density. As we can see, when the UE density is low, it is better to use the highest values (e.g., $b \approx 1$ ), while when the UE density is high, it is better to use the lowest values (in this case $b=0.3$ ). For each UE density, there is a value of $b$ that minimizes energy consumption; however, determining the exact value of the UE density is impossible in real scenarios. A simple solution would be to use the value of $b$ that covers the largest range of densities. From the figure, we observe that the optimal value of $b$ that covers the widest range of UE densities is between 0.3 and 0.5 . Thus, we choose $b=0.4$ as the parameter of our relaying mechanism.

\section{E. Comparison of the MTD Energy Consumption in Cellular Mode and D2D Mode}

In Fig. 12, we compare the energy consumed by the MTD when it uses cellular communication (cellular mode) and when it uses our relaying mechanism (D2D mode with $b=0.4$, $R_{d}=300$, and $W=16$ ). Fig. 12 (a) shows the average MTD energy consumption versus the MTD-BS distance $\left(d_{m, b}\right)$ and the UE density $\left(\lambda_{u}\right)$. We consider a scenario with $D=200$ bytes. It can be seen from the figure that for $\lambda_{u} \in[0.2 \times$ $\left.10^{-4}, 2 \times 10^{-4}\right] \mathrm{UEs} / \mathrm{m}^{2}$ and $d_{m, b}>500$ meters, the MTD energy consumption using our proposed mechanism is less than the energy consumption in cellular communication. We can also observe that in the same range of values, the MTDBS distance has little influence on the energy consumption in D2D mode. Besides, the MTD energy consumption is almost constant and does not depend on $\lambda_{u}$ for a large range of UE densities. This energy consumption is directly related to the relay discovery probability and the number of time slots used in the contention process. To analyze the behavior of relay discovery, we first define the average number of UEs per time slot $U_{s}$. To discover a relay only one time-slot with $U_{s}<$ 1 is required. When $U_{s}>1$, there is a high probability of collision. In the uniform case, all time-slots have the same $U_{s}$ value, which only depends on the UE density. When the density of UEs increases, all time-slots will have $U_{s}>1$ at a certain UE density. In the geometric case, $U_{s}$ depends on the UE density and the time-slot $s$. In the first time slots, $U_{s}$ is small and it increases as a function of $s$. When the UE density increases, the number of time slots with $U_{s}<1$ decreases. Nevertheless, for a large range of UE densities, there will be at least one time-slot with $U_{s}<1$ and thus a low probability of collision. In Fig. 12 (b), we compare the average MTD energy consumption versus the MTD-BS distance $\left(d_{m, b}\right)$ and the data packet size $(D)$. We consider a scenario with $\lambda_{u}=$ $1 \times 10^{-4} \mathrm{UEs} / \mathrm{m}^{2}$. In the figure, we show that our proposed relaying mechanism allows reducing the energy consumption significantly when the data packet size is 200 bytes and the MTD is far from the BS. We can also observe that in cellular and D2D modes, the energy consumption is linearly increasing with increasing data size. In cellular mode, $\bar{E}_{\text {cell }}$ is directly proportional to $D$, while in D2D mode $\bar{E}_{\mathrm{D} 2 \mathrm{D} \text {,total }}$ is the sum of two terms $\bar{E}_{\mathrm{D} 2 \mathrm{D} \text {,disc }}$ and $\bar{E}_{\mathrm{dataTx}}$. While in the discovery phase $\bar{E}_{\mathrm{D} 2 \mathrm{D} \text {, disc }}$ does not depends on $D$, in the data transmission phase $\bar{E}_{\text {dataTx }}$ is directly proportional to $D$.

We define the energy reduction factor as the ratio between the MTD energy consumption in D2D mode and the energy consumption in cellular mode. Table II presents the energy reduction factor for different values of $\lambda_{u}$ and $d_{m, b}$. We observe that in D2D mode for $\lambda_{u}=1 \times 10^{-4} \mathrm{UEs} / \mathrm{m}^{2}$, and $D=200$ bytes, the MTD consumes $23 \%$ and $75 \%$ less energy than in cellular mode for $d_{m, b}=500$ meters and $d_{m, b}=$ 1000 meters, respectively. In Table III, we present the energy reduction factor for data packets of different sizes and for two MTD-BS distances. We observe that in D2D mode for $d_{m, b}=1000$ meters, the MTD consumes $32 \%$ and $60 \%$ less energy than in cellular mode for $D=50$ and $D=100$ bytes, respectively.

\section{F. Comparison of Energy Consumption with Other Distributed Mechanisms}

We compare the performance of our relaying mechanism with the mechanism based on a uniform random choice of time-slot (Uniform) proposed in [10], and a baseline nonadaptive system $(N-A S)$ proposed in [18]. In the Uniform and 

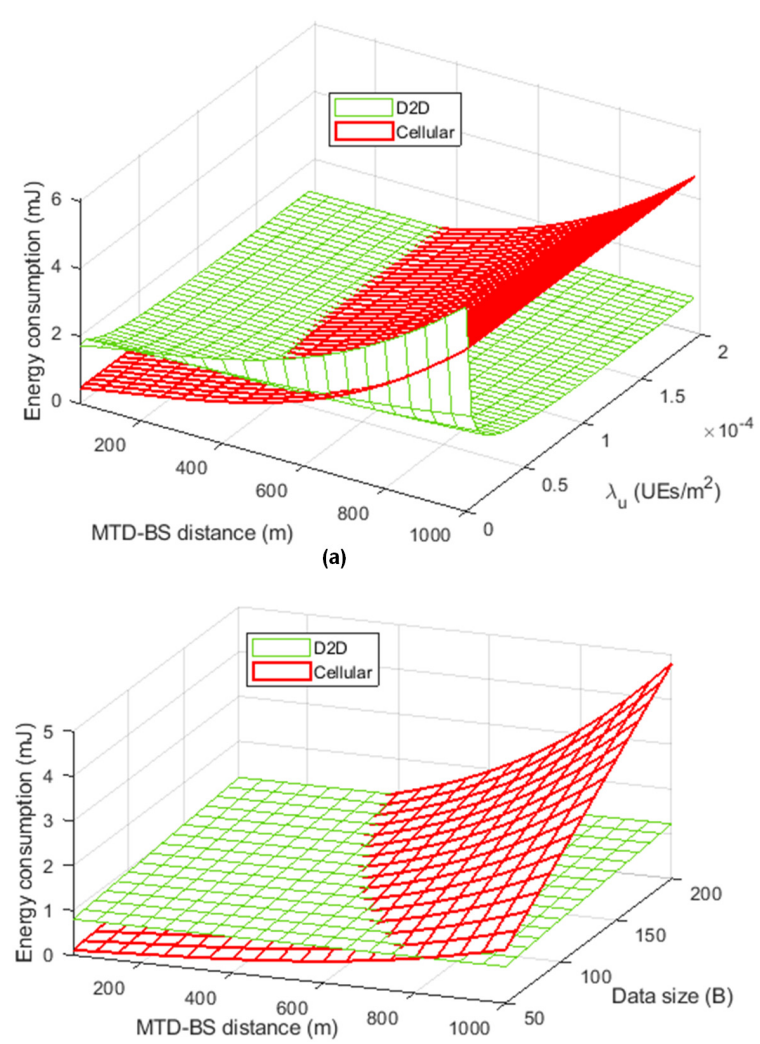

(b)

Fig. 12. Comparison of the total MTD energy consumption between cellular mode and D2D mode $\left(R_{d}=300, b=0.4\right.$, and $\left.W=16\right)$, shadowing $\sigma_{d B}=8 \mathrm{~dB}$. Average MTD energy consumption versus (a) MTD-BS distance and UE density for $D=200$ bytes and (b) MTD-BS distance and the data packet size for $\lambda_{u}=1 \times 10^{-4} \mathrm{UEs} / \mathrm{m}^{2}$.

TABLE II

COMPARISON OF THE ENERGY REDUCTION FACTOR FOR DIFFERENT $\lambda_{u}$ AND $d_{m, b}$ VALUES

\begin{tabular}{|c|c|c|c|c|}
\hline UE density (UEs/m $^{2}$ ) & \multicolumn{2}{|c|}{$\mathbf{0 . 5} \times \mathbf{1 0}^{-\mathbf{4}}$} & \multicolumn{2}{|c|}{$\mathbf{1} \times \mathbf{1 0}^{-\mathbf{4}}$} \\
\hline MTD-BS distance $(\mathbf{m})$ & $\mathbf{5 0 0}$ & $\mathbf{1 0 0 0}$ & $\mathbf{5 0 0}$ & $\mathbf{1 0 0 0}$ \\
\hline \hline Energy consumption D2D (mJ) & 1.30 & 1.36 & 1.20 & 1.22 \\
\hline Energy consumption Cellular $(\mathrm{mJ})$ & 1.55 & 4.80 & 1.55 & 4.80 \\
\hline Energy Reduction Factor & 0.84 & 0.28 & 0.77 & 0.25 \\
\hline
\end{tabular}

TABLE III

COMPARISON OF THE ENERGY REDUCTION FACTOR FOR DIFFERENT $D$ AND $d_{m, b}$ VALUES

\begin{tabular}{|c|c|c|c|c|}
\hline Data size (bytes) & \multicolumn{2}{|c|}{$\mathbf{5 0}$} & \multicolumn{2}{c|}{$\mathbf{1 0 0}$} \\
\hline MTD-BS distance $(\mathbf{m})$ & $\mathbf{5 0 0}$ & $\mathbf{1 0 0 0}$ & $\mathbf{5 0 0}$ & $\mathbf{1 0 0 0}$ \\
\hline \hline Energy consumption D2D (mJ) & 0.81 & 0.82 & 0.94 & 0.95 \\
\hline Energy consumption Cellular $(\mathrm{mJ})$ & 0.39 & 1.20 & 0.77 & 2.40 \\
\hline Energy reduction factor & 2.08 & 0.68 & 1.22 & 0.40 \\
\hline
\end{tabular}

$N-A S$ mechanisms, the MTD sends an RR packet and the UEs respond in a time-slot that is chosen randomly based on a uniform distribution. However, while in the Uniform mechanism the contention process ends as soon as the MTD correctly receives a response, in the $N-A S$ mechanism the MTD listens for the entire contention window and then selects the best relay (i.e., the UE with the lowest path loss) among all
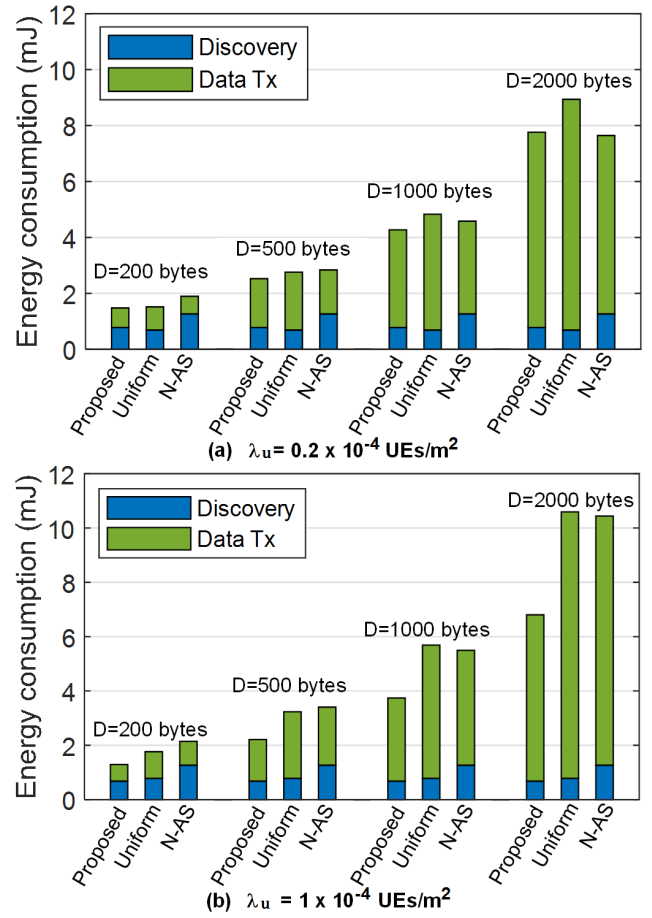

Fig. 13. Comparison of energy consumption between our proposed mechanism with $b=0.6$, Uniform [10], and N-AS [18], for $R_{d}=300, W=16$, shadowing $\sigma_{d B}=8 \mathrm{~dB}$, and $d_{m, b}=1000$ meters.

UEs that successfully responded to the MTD (i.e., without collisions).

Fig. 13 compares numerical results for two different UE densities (a) $\lambda_{u}=0.2 \times 10^{-4} \mathrm{UEs} / \mathrm{m}^{2}$ and (b) $\lambda_{u}=1 \times 10^{-4}$ $\mathrm{UEs} / \mathrm{m}^{2}$. The figure shows that in all cases, both our mechanism and the Uniform mechanism consume less energy than $N$ $A S$ during the discovery phase. This is because the contention process is stopped as soon as an RC packet is successfully received, while the $N-A S$ mechanism monitors the channel during all the contention window. As shown in Fig. 13 (a), the $N$-AS mechanism consumes less energy than our mechanism for a low UE density and long packets (e.g., 2000 bytes) because the collision probability is low and $N-A S$ really selects the best relay among all UEs. The energy saving in the transmission phase due to a higher bit rate is larger than the extra-energy spent by a longer discovery process. Fig. 13 (b) shows that for high UE densities our mechanism consumes less energy than both the Uniform mechanism and $N-A S$ during the data transmission phase. This is because our mechanism is less affected by high UE densities. In the case of Uniform mechanism and $N-A S$, there is a very low probability that the UE with the lowest path loss successfully responds to the MTD due to a high number of collisions. Furthermore, when the number of collisions is high, there is a greater probability with N-AS that the MTD correctly receives responses from distant UEs rather than from close UEs since the former are more numerous. 
TABLE IV

UE ENERGY CONSUMPTION IN THE DISCOVERY PHASE, FOR $R_{d}=300$ $b=0.6, W=16$.

\begin{tabular}{|c|c|c|c|c|}
\hline & \multicolumn{2}{|c|}{ RC is sent } & \multicolumn{2}{c|}{ RC is not sent } \\
\hline \hline UE density $\left(\mathrm{UEs} / \mathrm{m}^{2}\right) \times 10^{-4}$ & $\mathbf{0 . 2}$ & $\mathbf{2}$ & $\mathbf{0 . 2}$ & $\mathbf{2}$ \\
\hline Energy consumption $(\mathrm{mJ})$ & 0.60 & 0.55 & 0.33 & 0.28 \\
\hline
\end{tabular}

\section{G. UE Energy Consumption Cost}

In the discovery phase, the relay candidates can be divided into two groups: (i) UEs that send an RC packet and (ii) UEs that wait their turn to respond. In our mechanism, the MTD sends a feedback packet as soon as it finds a relay. The feedback packet carries the ID of the selected relay so that the non-selected UEs stop monitoring the channel. Therefore, in the first group the average energy consumption is $T_{s}\left(P_{m, T}+(\bar{S}+2) P_{m, R}\right)$ while in the second group the average energy consumption is $T_{s}(\bar{S}+2) P_{m, R}$, where $\bar{S}$ is the average number of time slots used in the contention process. The selected relay is part of the first group.

Table IV shows the UE energy consumption during the discovery phase for $R_{d}=300, b=0.6, W=16$, and $\lambda_{u}=\left\{0.2 \times 10^{-4}, 2 \times 10^{-4}\right\} \mathrm{UEs} / \mathrm{m}^{2}$. A UE that sends an $\mathrm{RC}$ packet consumes on average less than $0.6 \mathrm{~mJ}$ for a large range of UE densities. This energy consumption is negligible compared to the energy consumed by a UE, for example, when it is used to watch a video streaming [36]. During the data transmission phase, only the selected relay remains active. The energy consumed by the relay in this phase depends on the UE-BS parameters such as the MTD data, the transmission power, and the data rate. Nevertheless, this increase in energy consumption could be small if the approach proposed in [13] is adopted. In this approach, a UE aggregates the MTD data and supplement it with their own data, and transmits it to the BS.

Compared to traditional cellular communication, the D2D relaying mechanism allows a reduction of the MTD energy consumption. The cost of this reduction is an increase in the energy consumption of the UEs participating in the process. To reduce the UE energy consumption, we consider that the UEs will not be continuously monitoring the channel but only in a specified period known by the MTDs. On the other hand, when the direct link (MTD-BS) is under unfavorable propagation conditions, the MTD transmits its data for a long period if it uses the repetition technique [37], increasing the energy consumption, the delay, and the number of radio resources. Therefore, our mechanism not only allows to reduce energy consumption but also the delay and the consumption of radio resources. Nevertheless, it is worth mentioning that mMTC applications are delay tolerant.

\section{CONCLUSION AND FUTURE WORK}

In this paper, we propose a distributed D2D relaying mechanism, which is suitable for mMTC applications since it allows a reduction of the energy consumed by the MTD using a D2D relay mechanism. The key idea of our protocol is to use a contention process based on a truncated geometric distribution, which gives priority to UEs close to the MTD to be selected as relays. We show that using this mechanism, the MTD energy consumption is significantly reduced (up to $75 \%$ compared to direct cellular transmission). Moreover, our mechanism provides almost constant energy consumption for a large range of UE densities. Using stochastic geometry, we derive analytical expressions for the discovery probability, the average number of time slots used in the contention process, the PDF of the MTD-relay distance, and the total energy consumed by the MTD. These analytical expressions agree well with the simulation results.

An extension of this work is to optimize some parameters such as the discovery area and the contention window size. Further, this work can be extended to the scenario where the MTD is out-of-coverage. In this scenario, the relay would have to act as a synchronization source.

\section{ACKNOWLEDGMENT}

This paper is part of and thereby supported by the Horizon 2020 project ONE5G (ICT-760809) receiving funds from the European Union. The authors would like to acknowledge the contributions of their colleagues in the project, although the views expressed in this contribution are those of the authors and do not necessarily represent the project.

\section{REFERENCES}

[1] M. Series, "IMT Vision-Framework and overall objectives of the future development of IMT for 2020 and beyond," Recommendation ITU, pp. 2083-0, 2015.

[2] S. Parkvall, E. Dahlman, A. Furuskar, and M. Frenne, "Nr: The new $5 \mathrm{~g}$ radio access technology," IEEE Communications Standards Magazine, vol. 1, no. 4, pp. 24-30, 2017.

[3] V. W. Wong, Key technologies for $5 G$ wireless systems. Cambridge university press, 2017.

[4] 3GPP, "Feasibility study for Proximity Services (ProSe)," 3rd Generation Partnership Project (3GPP), Technical Report (TR) 23.803, 2013.

[5] K. Vanganuru, S. Ferrante, and G. Sternberg, "System capacity and coverage of a cellular network with $\mathrm{d} 2 \mathrm{~d}$ mobile relays," in MILCOM 2012-2012 IEEE Military Communications Conference. IEEE, 2012, pp. $1-6$.

[6] G. Zhao, S. Chen, L. Qi, L. Zhao, and L. Hanzo, "Mobile-traffic-aware offloading for energy-and spectral-efficient large-scale d2d-enabled cellular networks," IEEE Transactions on Wireless Communications, 2019.

[7] H. Zhang, L. Song, and Y. J. Zhang, "Load balancing for 5g ultra-dense networks using device-to-device communications," IEEE Transactions on Wireless Communications, vol. 17, no. 6, pp. 4039-4050, 2018.

[8] U. Tefek and T. J. Lim, "Relaying and radio resource partitioning for machine-type communications in cellular networks," IEEE Transactions on Wireless Communications, vol. 16, no. 2, pp. 1344-1356, 2016.

[9] R. Ma, Y.-J. Chang, H.-H. Chen, and C.-Y. Chiu, "On relay selection schemes for relay-assisted d2d communications in lte-a systems," IEEE Transactions on Vehicular Technology, vol. 66, no. 9, pp. 8303-8314, 2017.

[10] C. V. Anamuro, N. Varsier, J. Schwoerer, and X. Lagrange, "Energyefficient discovery process for mmtc applications," in 2019 12th IFIP Wireless and Mobile Networking Conference (WMNC), Sep. 2019, pp. 79-86.

[11] Y. Li, C. Liao, Y. Wang, and C. Wang, "Energy-efficient optimal relay selection in cooperative cellular networks based on double auction," IEEE Transactions on Wireless Communications, vol. 14, no. 8, pp. 4093-4104, 2015.

[12] H. Nishiyama, M. Ito, and N. Kato, "Relay-by-smartphone: realizing multihop device-to-device communications," IEEE Communications Magazine, vol. 52, no. 4, pp. 56-65, 2014.

[13] G. Rigazzi, N. K. Pratas, P. Popovski, and R. Fantacci, "Aggregation and trunking of $\mathrm{m} 2 \mathrm{~m}$ traffic via d2d connections," in 2015 IEEE international conference on communications (ICC). IEEE, 2015, pp. 2973-2978. 
[14] H. Zhang, B. Di, K. Bian, and L. Song, "Iot-u: Cellular internet-of-things networks over unlicensed spectrum," IEEE Transactions on Wireless Communications, vol. 18, no. 5, pp. 2477-2492, 2019.

[15] A. Al-Hourani, S. Kandeepan, and E. Hossain, "Relay-assisted deviceto-device communication: A stochastic analysis of energy saving," IEEE Transactions on Mobile Computing, vol. 15, no. 12, pp. 3129-3141, 2016.

[16] M. N. Tehrani, M. Uysal, and H. Yanikomeroglu, "Device-to-device communication in $5 \mathrm{~g}$ cellular networks: challenges, solutions, and future directions," IEEE Communications Magazine, vol. 52, no. 5, pp. 86-92, 2014.

[17] G. Liu, F. R. Yu, H. Ji, V. C. Leung, and X. Li, "In-band full-duplex relaying: A survey, research issues and challenges," IEEE Communications Surveys \& Tutorials, vol. 17, no. 2, pp. 500-524, 2015.

[18] V. Shah, N. B. Mehta, and R. Yim, "The relay selection and transmission trade-off in cooperative communication systems," IEEE Transactions on Wireless Communications, vol. 9, no. 8, pp. 2505-2515, 2010.

[19] M. Seyfi, S. Muhaidat, J. Liang, and M. Dianati, "Effect of feedback delay on the performance of cooperative networks with relay selection," IEEE Transactions on Wireless Communications, vol. 10, no. 12, pp. 4161-4171, 2011.

[20] D. S. Michalopoulos, H. A. Suraweera, G. K. Karagiannidis, and R. Schober, "Amplify-and-forward relay selection with outdated channel estimates," IEEE Transactions on Communications, vol. 60, no. 5, pp. 1278-1290, 2012.

[21] Z. Zhou, S. Zhou, J.-H. Cui, and S. Cui, "Energy-efficient cooperative communication based on power control and selective single-relay in wireless sensor networks," IEEE transactions on wireless commun. vol. 7, no. 8, pp. 3066-3078, 2008.

[22] A. Bletsas, H. Shin, and M. Z. Win, "Cooperative communications with outage-optimal opportunistic relaying," IEEE Transactions on Wireless Communications, vol. 6, no. 9, pp. 3450-3460, 2007.

[23] F. Ouyang, J. Ge, F. Gong, and J. Hou, "Collision resolving relay selection in large-scale blind relay networks," Wireless Networks, vol. 23, no. 6, pp. 1793-1807, 2017.

[24] A. Alsharoa, X. Zhang, D. Qiao, and A. Kamal, "An energy-efficient relaying scheme for internet of things communications," in 2018 IEEE International Conference on Communications (ICC). IEEE, 2018, pp. $1-6$.

[25] A. Awang, X. Lagrange, and D. Ros, "Toward an analysis of energy consumption in multihop wireless sensor networks," in 2010 International Conference on Intelligent and Advanced Systems. IEEE, 2010 pp. 1-6.

[26] J. Chai, L. Feng, F. Zhou, P. Zhao, P. Yu, and W. Li, "Energyefficient resource allocation based on hypergraph $3 \mathrm{~d}$ matching for $\mathrm{d} 2 \mathrm{~d}$ assisted mmtc networks," in 2018 IEEE Global Commun. Conference (GLOBECOM). IEEE, 2018, pp. 1-7.

[27] H. Feng, Y. Xiao, and L. J. Cimini, "Net throughput of centralized and decentralized cooperative networks with relay selection," IEEE Wireless Communications Letters, vol. 3, no. 5, pp. 477-480, 2014

[28] A. Awang, X. Lagrange, and D. Ros, "Rssi-based forwarding for multihop wireless sensor networks," in Meeting of the European Network of Universities and Companies in Information and Commun. Engineering. Springer, 2009, pp. 138-147.

[29] H. S. Dhillon and J. G. Andrews, "Downlink rate distribution in heterogeneous cellular networks under generalized cell selection," IEEE Wireless Communications Letters, vol. 3, no. 1, pp. 42-45, 2013.

[30] Q. Song, L. Nuaymi, and X. Lagrange, "Analysis of macro diversity based on maximum ratio combining in long range aloha networks," Telecommunication Systems, pp. 1-12, 2019.

[31] 3GPP, "Cellular system support for ultra low complexity and low throughput internet of things," 3rd Generation Partnership Project (3GPP), Technical Report (TR) 45.820, 2015, version 13.1.0.

[32] S. Ahmadi, LTE-Advanced: a practical systems approach to understanding $3 G P P$ LTE releases 10 and 11 radio access technologies. Academic Press, 2013.

[33] R. L. Streit, Poisson point processes: imaging, tracking, and sensing. Springer Science \& Business Media, 2010.

[34] 3GPP, "Evolved Universal Terrestrial Radio Access (E-UTRA); Radio Frequency (RF) system scenarios," 3rd Generation Partnership Project (3GPP), Technical Report (TR) 36.942, 06 2018, version 15.0.0.

[35] P. Mogensen, W. Na, I. Z. Kovács, F. Frederiksen, A. Pokhariyal, K. I. Pedersen, T. Kolding, K. Hugl, and M. Kuusela, "Lte capacity compared to the shannon bound," in 2007 IEEE 65th vehicular technology conference-VTC2007-Spring. IEEE, 2007, pp. 1234-1238.

[36] R. Trestian, A.-N. Moldovan, O. Ormond, and G.-M. Muntean, "Energy consumption analysis of video streaming to android mobile devices," in
2012 IEEE Network Operations and Management Symposium. IEEE, 2012, pp. 444-452.

[37] C. Bockelmann, N. K. Pratas, G. Wunder, S. Saur, M. Navarro, D. Gregoratti, G. Vivier, E. De Carvalho, Y. Ji, Č. Stefanović et al., "Towards massive connectivity support for scalable mmtc communications in $5 \mathrm{~g}$ networks," IEEE access, vol. 6, pp. 28 969-28 992, 2018.

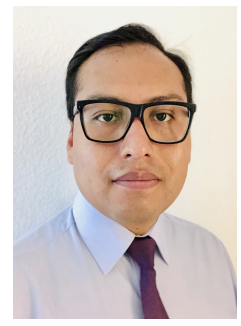

Cesar Vargas Anamuro received the Telecommunications engineer diploma from the National University of Engineering of Lima, Peru in 2012. He received the Master of Information Technology from IMT Atlantique-Brest, France in 2015 and his Ph.D. degree in 2020. He is currently working as a postdoc researcher at IMT Atlantique-Rennes, France. His research interests include cellular networks and D2D communication schemes for mMTC applications.

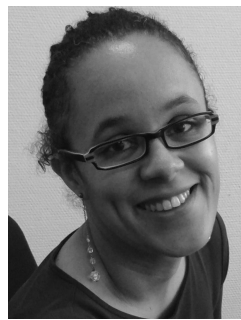

Nadège Varsier received the Master of engineering from IMT Atlantique-Brest in 2002 and the Ph.D. degree in Electrical and Electronic Engineering from the Tokyo Metropolitan University, Japan, in 2009. At Orange, France since 2010, her actual researches are dedicated to IoT networks. She has been involved in many collaborative research projects such as the EU FP7 LEXNET project or the 5GPPP Fantastic 5G and ONE5G projects. She has authored and coauthored more than 40 scientific papers in peerreviewed journals and contributions to international

conferences.

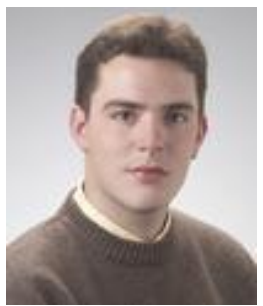

Jean Schwoerer received an Engineering degree in electronic and communication systems from the National Institute of Applied Sciences (INSA) in Rennes in 2002 and the $\mathrm{PhD}$ degree in electronic in 2006 from University of Rennes. His pursuing work at Orange Labs Grenoble leads him to focus on low power radio interfaces for the Internet of Things, on which he is leading Orange research activities. This includes "alternative" radio technologies like LPWA or mesh network as well as mobile network technologies (4G and $5 \mathrm{G}$ ). Additionally, as Orange delegate to IEEE 802, ETSI and then 3GPP RAN, he wears many contributions in standardization on those two topics for the Orange group.

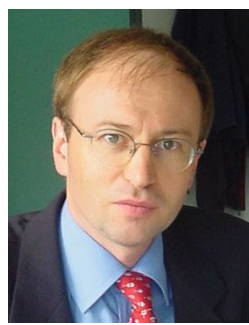

Xavier Lagrange received the Engineering Degree from Ecole Centrale des Arts et Manufactures, Paris, France, in 1984 and the Ph.D. degree from TELECOM Paristech in 1998. Since 2002, he has been professor in IMT Atlantique (formerly known as Telecom Bretagne) at the "Network Systems, Cybersecurity and Digital Law". He leads research group ADOPNET (Advanced Technologies for Operated Networks) at IRISA (Institut de Recherche en Informatique et Systèmes Aléatoires). His domain of interest includes resource allocation, medium access control and performance analysis for $4 \mathrm{G}$ and $5 \mathrm{G}$ cellular networks. He is a senior member of IEEE. 